\title{
Engagement of TNF mRNA by an endotoxin-inducible cytoplasmic protein
}

\author{
Cyril Gueydan, Laurent Houzet, Arnaud Marchant, André Sels, \\ Georges Huez and Véronique Kruys
}

Molecular Medicine 2, 479-488 (1996)

Some of the data in Fig. 1B was reported incorrectly. The correct version is presented below:

The AU + RNA probe previously described as being 70 nucleotides long is actually composed of 135 nucleotides. This is due to the presence of plasmid sequences located between the RNA polymerase promoter and the AU-rich sequence.

This AU+ RNA probe does not form complex 2 with cytoplasmic extracts of LPS-stimulated macrophages. By performing experiments to define more precisely the RNA sequence involved in the formation of the LPS-induced RNA-protein complex 2, we discovered that a RNA probe composed strictly of the AU-rich sequence forms complex 2. This result indicates that the RNA sequence involved in complex 2 formation is located within the AU-rich sequence. The absence of binding by the AU + probe might, therefore, result from change in the RNA conformation due to the presence of plasmid sequence in the probe. Alternatively, the binding of irrelevant protein(s) on the plasmid sequences might prevent the formation of complex 2 .

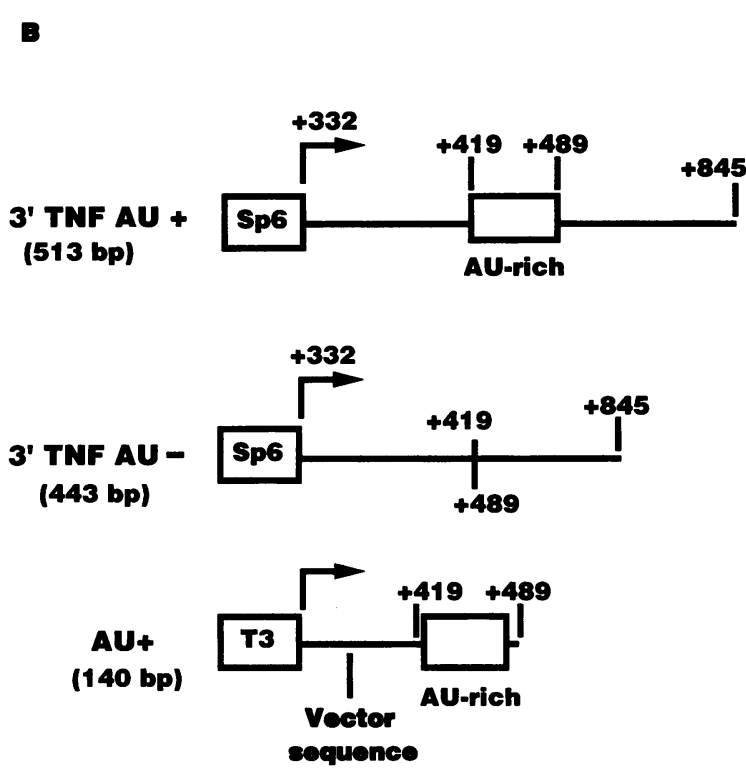

FIG. 1 B.

Schematic representation of the plasmid templates used for in vitro transcription of the RNA probes. 